\section{NET formation implicated in myositis}

A distinct subset of neutrophils called low-density granulocytes (LDGs) contribute to the pathogenesis of a number of systemic autoimmune diseases, including systemic lupus erythematosus, in part through the formation of neutrophil extracellular traps (NETs). New findings implicate aberrant NET formation by LDGs in idiopathic inflammatory myopathies (IIM).

"We found that the presence of LDGs and NETs associates with clinical features of IIM and that NETs are elevated in the circulation and various organs of patients with IIM," reports Mariana Kaplan, corresponding author on the new study. Increased levels of circulating NETs were associated with disease activity as well as with the presence of certain myositis-associated antibodies - in particular, antibodies against melanoma differentiation-associated protein 5 (MDA5). Notably, treatment of normal-density granulocytes with anti-MDA5 antibodies isolated from patients with IIM promoted NET formation in vitro. ing analysis identified a neutrophil gene signature in the skeletal muscle of patients with dermatomyositis (a subtype of IIM) that was associated with the expression of type I and type II interferon-regulated genes as well as indicators of muscle damage.

To investigate the pathogenic role of NETs in IIM, the researchers
RNA sequenc- looked at the effects of NETs on muscle myotubes (formed from precursor myoblasts in vitro). NETs isolated from patients with IIM impaired the viability of the skeletal myotubes in vitro, but did not reduce myoblast proliferation.

The damaging in vitro effects of NETs were mediated in part by citrullinated histones (a component of NETs) and could be partially inhibited with an anti-histone $\mathrm{H} 4$ antibody. Importantly, treatment with citrullinated $\mathrm{H} 4$, but not treatment with native $\mathrm{H} 4$, was toxic to myotubes, recapitulating the effects of NETs in vitro.

"Our results implicate neutrophil dysregulation in the pathogenesis of adult and childhood-onset IIM and suggest that targeting pathways of NET formation could have therapeutic roles in this disease," concludes Kaplan.

Jessica McHugh

ORIGINAL ARTICLE Seto, N. L. et al. Neutrophil dysregulation is pathogenic in idiopathic inflammatory myopathies. JCl Insight https:// doi.org/10.1172/jci.insight.134189 (2020)

\title{
LDL subfraction linked to vascular ageing and heart disease in SLE
}

Patients with systemic lupus erythematosus (SLE) have signs of early vascular ageing and an increased risk of cardiovascular disease, but do not have increased concentrations of low-density lipoprotein (LDL) in their blood compared with healthy individuals. LDL can be separated into five increasingly electronegative subfractions (L1-L5), of which $L 5$ is known to be atherogenic. For the first time, researchers have examined the $L 5$ subfraction from patients with SLE and investigated its role in SLE-associated cardiovascular changes. In the new study, patients with SLE had less total plasma LDL than healthy individuals but a higher percentage of the $L 5$ subfraction, which correlated with clinical measures of vascular ageing. The researchers tested the atherogenic properties of LDL from patients with SLE by injecting it into $\mathrm{Apoe}^{-/-}$ mice. These atherosclerosis-prone mice developed signs of vascular ageing and atherosclerosis in response to $L D L$ from patients with SLE, but not in response to LDL from healthy individuals.

"We found a high content of lysophosphatidylcholine and platelet-activating factor in the total LDL preparations from patients with SLE, especially in the $L 5$ subfractions," say corresponding authors Liang-Yin Ke, Jeng-Hsien Yen and Chu-Huang Chen. "These inflammatory mediators can lead to the differentiation of monocytes into pro-inflammatory $\mathrm{CD}_{16}{ }^{+}$cells."

$\mathrm{CD} 16^{+}$monocytes were present at increased numbers in the blood of patients with SLE compared with healthy individuals. Interestingly, both plasma concentrations of the chemokine $\mathrm{CX}_{3} \mathrm{CL} 1$ and expression of its receptor $\mathrm{CX}_{3} \mathrm{CR} 1$ on $\mathrm{CD} 16^{+}$monocytes were also increased in patients with SLE compared with healthy individuals.

"Through $\mathrm{CX}_{3} \mathrm{CL} 1-\mathrm{CX}_{3} \mathrm{CR} 1$ interactions, $\mathrm{CD} 16^{+}$monocytes adhere

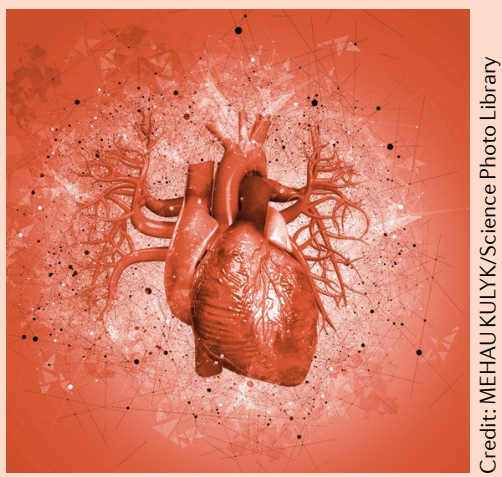

to the damaged endothelium, which facilitates their penetration into the subendothelium," explains first author Hua-Chen Chan. "This cascade of events, induced by L5 LDL, contributes to endothelial dysfunction and vascular ageing, thereby providing a novel explanation for the early onset of atherosclerosis-associated complications in patients with SLE," she concludes.

\section{Joanna Clarke}

ORIGINAL ARTICLE Chan, H.-C. et al. Role of low-density lipoprotein in early vascular aging associated with systemic lupus erythematosus. Arthritis Rheumatol. https://doi.org/10.1002/ art.41213 (2020) 